\title{
ON THE CONSTRUCTION OF SETS OF MUTUALLY ORTHOGONAL LATIN SQUARES AND THE FALSITY OF A CONJECTURE OF EULER( $\left.{ }^{1}\right)$
}

\author{
BY
}

\author{
R. C. BOSE AND S. S. SHRIKHANDE
}

1. Introduction. If $p_{1}^{n_{1}} p_{2}^{n_{2}} \cdots p_{u^{u}}^{n_{4}}$ is the prime power decomposition of an integer $v$, and we define the arithmetic function $n(v)$ by

$$
n(v)=\min \left(p_{1}^{n_{1}}, p_{2}^{n_{2}}, \cdots, p_{u}^{n_{u}}\right)-1
$$

then it is known, MacNeish [16] and Mann [17] that there exists a set of at least $n(v)$ mutually orthogonal Latin squares (m.o.l.s.) of order $v$. It seemed plausible that $n(v)$ is also the maximum possible number of m.o.l.s. of order $v$. This would have implied the correctness of Euler's [13] conjecture about the nonexistence of two orthogonal Latin squares of order $v$ when $v=2(\bmod 4)$, since $n(v)=1$ in this case. If we denote by $N(v)$ the maximum possible number of mutually orthogonal Latin squares of order $v$, then Parker [18] showed that in certain cases $N(v)>n(v)$ by proving that if there exists a balanced incomplete block (BIB) design with $v$ treatments, $\lambda=1$, and block size $k$ which is a prime power then $N(v) \geqq k-2$. This result cast a doubt on the validity of Euler's conjecture. In this paper we generalize Parker's method of constructing m.o.l.s., by showing that a very general class of designs, which we have called pairwise balanced designs of index unity, can be used for the construction of sets of m.o.l.s. Various applications of this method have been made. In particular we show that Euler's conjecture is false for an infinity of values of $v$, including all values of the form $36 m+22$. We also provide a table for all values of $v \leqq 150$, for which our methods enable us to show that $N(v)>n(v)$. The smallest number for which we have been able to demonstrate the falsity of Euler's conjecture is 22. Two orthogonal squares of order 22 constructed by our method are given in the Appendix. Several attempts, necessarily erroneous, have been made in the past to prove Euler's conjecture, e.g., Peterson [19], Wernicke [20] and MacNeish [16]. Levi [14, p. 14] points

Presented to the Society on April 24, 1959 under the titles Note on Parker's method of constructing pairwise orthogonal sets of Latin squares by R. C. Bose and Group divisible designs and the construction of pairwise orthogonal sets of Latin squares by S. S. Shrikhande and on June 20, 1959 under the title $A$ theorem on the construction of orthogonal Latin squares and the falsity of Euler's conjecture by R. C. Bose and S. S. Shrikhande; received by the editors April 10, 1959.

(1) This research was supported by the United States Air Force through the Air Force Office of Scientific Research of the Air Research and Development Command, under Contract No. AF 49(638)-213. Reproduction in whole or in part is permitted for any purpose of the United States Government. 
out the inadmissibility of the argument used by Peterson and MacNeish. Wernicke's argument was shown to be fallacious by MacNeish [15].

2. Orthogonal Latin squares and orthogonal arrays. A Latin square of order $v$ may be defined as an arrangement of $v$ symbols, say $1,2, \cdots, v$ in a $v \times v$ square such that each symbol occurs once in every row and every column. Two Latin squares are said to be orthogonal, when if they are superposed each symbol of the first square occurs just once with each symbol of the second square. A set of mutually orthogonal Latin squares (m.o.l.s.) is a set of Latin squares any two of which are orthogonal.

Defining $N(v)$ and $n(v)$ as in the introduction, we have already referred to the MacNeish-Mann theorem viz. $N(v) \geqq n(v)$. We shall now give a useful lemma which generalizes this result.

Lemma 1. If $v=v_{1} v_{2} \cdots v_{u}$ then $N(v) \geqq \min \left(N\left(v_{1}\right), N\left(v_{2}\right), \cdots, N\left(v_{u}\right)\right)$.

We shall derive this lemma from a general result on orthogonal arrays due to Bush and one of the authors $[5 ; 10]$. Let us consider a matrix $A=\left(a_{i j}\right)$, where each $a_{i j}$ represents one of the symbols $1,2, \cdots, v$, with $m=\lambda v^{t}$ columns and $r$ rows. Consider all $t$ rowed submatrices which can be formed from $A$ by choosing $t$ rows, $t \leqq r$. Each column of the submatrix can be regarded as an ordered $t$-plet. The matrix $A$ is called an orthogonal array $(m, r, v, t)$ of size $m, r$ constraints, $v$ levels, strength $t$ and index $\lambda$; if each $t$ rowed submatrix which can be formed from $A$ contains every one of the $v^{t}$ possible ordered $t$-plets each repeated $\lambda$ times. If $f(m, v, t)$ denotes the maximum number of constraints for given $m, v$ and $t$, then the following theorem is known $[5 ; 10]$.

THEOREM. If $m_{i}$ is divisible by $v_{i}^{i}$ for $i=1,2, \cdots, u$, then

$$
f\left(m_{1} m_{2} \cdots m_{u}, v_{1} v_{2} \cdots v_{u}, t\right) \geqq \min \left(r_{1}, r_{2}, \cdots, r_{u}\right)
$$

where $r_{i}=f\left(m_{i}, v_{i}, t\right)$.

Now it is known [11] that an orthogonal array $\left(v^{2}, q, v, 2\right)$ of strength 2 and index unity is equivalent to the existence of a set of $q-2$ m.o.l.s. of order $v$, which may be obtained from this array by using two of the rows for coordinatization. Conversely the array can be obtained from the squares. Hence $f\left(v^{2}, v, 2\right)=N(v)+2$. The required lemma follows by taking $m_{i}=v_{i}^{2}, t=2$ in the theorem.

The MacNeish-Mann theorem follows from the lemma by taking $v_{i}=p_{i}^{n_{i}}, i=1,2, \cdots, u$. If $N(v)$ had always been equal to $n(v)$ the lemma would add nothing new. However this is not the case. For example it will be shown in $\S 5$, that $N(21) \geqq 4$. It follows from the lemma that $N(105)$ $\geqq \min (N(21), N(5)) \geqq 4$ whereas $n(105)=\min (3,5,7)-1=2$.

3. Pairwise balanced designs of index unity. An arrangement of $v$ objects (called treatments) in $b$ sets (called blocks) will be called a pairwise balanced 
design of index unity and type $\left(v ; k_{1}, k_{2}, \cdots, k_{m}\right)$ if each block contains either $k_{1}, k_{2}, \cdots$ or $k_{m}$ treatments which are all distinct $\left(k_{i} \leqq v, k_{i} \neq k_{j}\right)$, and every pair of distinct treatments occurs in exactly one block of the design. If the number of blocks containing $k_{i}$ treatments is $b_{i}$, then clearly

$$
b=\sum_{i=1}^{m} b_{i}, \quad v(v-1)=\sum_{i=1}^{m} b_{i} k_{i}\left(k_{i}-1\right) .
$$

Consider a pairwise balanced design $(D)$ of index unity and type $\left(v ; k_{1}, k_{2}, \cdots, k_{m}\right)$. The subdesign $\left(D_{i}\right)$ formed by the blocks of size $k_{i}$ will be called the $i$ th equiblock component of $(D), i=1,2, \cdots, m$.

A subset of blocks belonging to any equiblock component $\left(D_{i}\right)$ will be said to be of type I if every treatment occurs in the subset exactly $k_{i}$ times. The number of blocks in such a subset is clearly $v$. As noted by Levi using Konig's theorem on the decomposition of even regular graphs [14, pp. 4-6], we can rearrange the treatments within the blocks of the subset in such a way that every treatment comes in each position exactly once. If the $v$ blocks of the subset are written out as columns, each treatment occurs exactly once in every row. When so written out the blocks will be said to be in the standard form.

A subset of blocks belonging to any equiblock component $\left(D_{i}\right)$ will be said to be of type II if every treatment occurs in the subset exactly once. The number of blocks in such a subset is $v / k_{i}$.

The component $\left(D_{i}\right)$ will be defined to be separable if the blocks can be divided into subsets of type I or type II (both types may occur in $\left(D_{i}\right)$ at the same time). Let $r_{i}$ be the number of subsets of type I and $s_{i}$ the number of subsets of type II in $\left(D_{i}\right)$, then clearly

$$
v\left(r_{i} k_{i}+s_{i}\right)=k_{i} b_{i}
$$

The design $(D)$ is defined to be separable if each equiblock component $\left(D_{i}\right)$ is separable. It follows from (3.1) that

$$
v-1=\sum_{i=1}^{m}\left(r_{i} k_{i}+s_{i}\right)\left(k_{i}-1\right)
$$

4. Main theorem. We shall first prove the following:

Lemma 2. Suppose there exists a set $\Sigma$ of $q-1$ m.o.l.s. of order $k$, then we can construct a $q \times k(k-1)$ matrix $P$, whose elements are the symbols $1,2, \cdots, k$ and such that (i) any ordered pair $(i, j), i \neq j$ occurs as a column exactly once in any two rowed submatrix of $P$, (ii) $P$ can be subdivided into $k-1$ submatrices $P_{1}, P_{2}, \cdots, P_{k-1}$ of order $q \times k$ such that in each row of $P_{c}, 1 \leqq c \leqq k-1$, each of the symbols $1,2, \cdots, k$ occurs exactly once.

Proof. It has been shown in [9] that matrix $P$ with the property (i) can be obtained in the following manner. Without loss of generality we can take 
the set $\Sigma$ in a form in which the first row of each Latin square contains the symbols $1,2, \cdots, k$ in that order and prefix to the set $\Sigma$ a square containing the symbol $i$ in each position in the $i$ th column. If we then write the elements of each square in a single row such that the symbol in the $i$ th row and $j$ th column occupies the $n$th position in the row where $n=k(i-1)+j$, and from the resulting $q \times k^{2}$ matrix we delete the first $k$ columns, we get the matrix $P$. If $P_{c}$ is the submatrix of $P$ formed by the columns $k(c-1)+j, j=1,2, \cdots, k$, then the property (ii) follows from the fact that each of the symbols $1,2, \cdots, k$ appears exactly once in any given row of a Latin square belonging to $\Sigma$.

Let $\gamma$ be a column of $k$ distinct treatments chosen from the set $t_{1}, t_{2}, \cdots, t_{v}$ then following the notation used in [9], we shall denote by $P(\gamma)$, the $q \times k(k-1)$ matrix obtained from $P$ on replacing the symbol $i$ by the treatment occurring in the $i$ th position in $\gamma$. A similar meaning will be assigned to $P_{i}(\gamma)$ and $\pi_{c j}(\gamma)$ where $\pi_{c j}$ denotes the $j$ th column of $P_{c}$.

Clearly every treatment of $\gamma$ occurs once in every row of $P_{c}(\gamma), c=1,2$, $\cdots, k-1$, and if $t_{a}$ and $t_{b}$ are any two treatments of $\gamma$ then the ordered pair $\left(t_{a}, t_{b}\right)$ occurs as a column exactly once in any two rowed submatrix of $P(\gamma)$.

Theorem 1. Let there exist a pairwise balanced design $(D)$ of index unity and type $\left(v ; k_{1}, k_{2}, \cdots, k_{m}\right)$ and suppose there exist $q_{i}-1$ m.o.l.s. of order $k_{i}$. If

$$
q=\min \left(q_{1}, q_{2}, \cdots, q_{m}\right)
$$

then there exist at least $q-2$ m.o.l.s. of order $v$. If the design $(D)$ is separable then the number of m.o.l.s. of order $v$ is at least $q-1$.

Proof. The first part of the theorem was proved in [9]. However in order to prove the second part it will be necessary to reproduce this proof with slightly altered notation. Let the treatments of the design be $t_{1}, t_{2}, \cdots, t_{v}$, and let the blocks of the design (written out as columns) belonging to the equiblock component $\left(D_{i}\right)$ be $\delta_{i 1}, \delta_{i 2}, \cdots, \delta_{i b_{i}}(i=1,2, \cdots, m)$. We now define the $k_{i} \times b_{i}$ matrix $D_{i}$ by

$$
D_{i}=\left[\delta_{i 1}, \delta_{i 2}, \cdots, \delta_{i b_{i}}\right] \text {. }
$$

Let $P_{i}$ be the matrix of order $q_{i} \times k_{i}\left(k_{i}-1\right)$ defined in Lemma 2 , the elements of $P_{i}$ being the symbols $1,2, \cdots, k_{i}$. Let $P_{i c}, c=1,2, \cdots, k_{i}-1$ be the submatrices of $P_{i}$, such that each row of $P_{i c}$ contains the symbols $1,2, \cdots, k_{i}$ exactly once and let $\pi_{i c j}$ be the $j$ th column of $P_{i c}, j=1,2, \cdots, k_{i}$. Let $P_{i}\left(\delta_{i u}\right), u=1,2, \cdots, b_{i}$, be the matrix obtained from $P_{i}$ and $\delta_{i u}$, and let

$$
P_{i}\left(D_{i}\right)=\left[P_{i}\left(\delta_{i 1}\right), P_{i}\left(\delta_{i 2}\right), \cdots, P_{i}\left(\delta_{i b_{i}}\right)\right] .
$$

Then $P_{i}\left(D_{i}\right)$ is of order $q_{i} \times b_{i} k_{i}\left(k_{i}-1\right)$. If $t_{a}$ and $t_{b}$ are any two treatments occurring in the same block of $\left(D_{i}\right)$, then the ordered pair $\left(t_{a}, t_{b}\right)$ occurs exactly once as a column in any two rowed submatrix of $P_{i}\left(D_{i}\right)$. Let $\Delta_{i}$ be the matrix obtained from $P_{i}\left(D_{i}\right)$ by retaining only the first $q$ rows, and let 


$$
\Delta=\left[\Delta_{1}, \Delta_{2}, \cdots, \Delta_{m}\right] .
$$

Then from (3.1), $\Delta$ is of order $q \times v(v-1)$, and it follows from the properties of the design that any two rowed submatrix of $\Delta$ contains as a column each ordered pair of two distinct treatments chosen from $t_{1}, t_{2}, \cdots, t_{v}$ exactly once. This property of $\Delta$ will be referred to as the property $\tau_{2}$.

Let $\Delta_{0}$ be a $q \times v$ matrix whose $i$ th column contains $t_{i}$ in every position $(i=1,2, \cdots, v)$. Then from [11] the matrix $\left[\Delta_{0}, \Delta\right]$ is an orthogonal array $\left[v^{2}, q, v, 2\right]$ of order $v^{2}, q$ constraints, $v$ levels and strength 2 . Using two rows to coordinatize we get a set of $q-2$ m.o.l.s. which proves the first part of the theorem.

To prove the second part of our theorem we shall introduce the concept "column equivalence." Two matrices $A$ and $B$ will be called column equivalent if they can be obtained from each other by a rearrangement of columns. This relation will be denoted by $A \sim B$.

Suppose now that the design $(D)$ is separable. Let $\left(D_{i 1}\right), \cdots,\left(D_{i r_{i}}\right)$ be the subsets of type I, and $\left(D_{i 1}^{*}\right), \cdots,\left(D_{s_{i}}^{*}\right)$ be the subsets of type II into which the blocks of $\left(D_{i}\right)$ are divisible, the blocks of subsets of type I being written in the standard form. We shall show that after a rearrangement of columns, $\Delta$ can be divided into submatrices of order $q \times v$, possessing the property $\tau_{1}$ that each row contains each of the treatments $t_{1}, t_{2}, \cdots, t_{v}$ exactly once. Now

$$
P_{i}\left(D_{i f}\right) \sim\left[\cdots, \pi_{i c j}\left(D_{i f}\right), \cdots\right], \quad c=1,2, \cdots, k_{i}-1, j=1,2, \cdots, k_{i} .
$$

Since $\pi_{i c j}\left(D_{i f}\right), f=1,2, \cdots, r_{i}$, clearly possesses the property $\tau_{1}, P_{i}\left(D_{i f}\right)$ is divisible (up to a rearrangement of columns) into $k_{i}\left(k_{i}-1\right)$ submatrices each having the property $\tau_{1}$.

Again

$$
P_{i}\left(D_{i g}^{*}\right) \sim\left[\cdots, P_{i c}\left(D_{i g}^{*}\right), \cdots\right], \quad c=1,2, \cdots, k_{i}-1 .
$$

Since $P_{i c}\left(D_{i g}^{*}\right), g=1,2, \cdots, s_{i}$, possesses the property $\tau_{1}, P_{i}\left(D_{i g}^{*}\right)$ is divisible (up to a rearrangement of columns) into $k_{i}-1$ submatrices each with the property $\tau_{1}$. Since

$$
P_{i}\left(D_{i}\right)=\left[P_{i}\left(D_{i 1}\right), \cdots, P_{i}\left(D_{i r_{i}}\right), P_{i}\left(D_{i 1}^{*}\right), \cdots, P_{i}\left(D_{i s_{i}}^{*}\right)\right]
$$

and since the property $\tau_{1}$ remains unchanged by deletion of rows it follows from (3.3) that after a rearrangement of columns $\Delta$ can be divided into $v-1$ submatrices $\Delta^{(1)}, \Delta^{(2)}, \cdots, \Delta^{(v-1)}$ each with the property $\tau_{1}$. It should also be noted that the property $\tau_{2}$ of $\Delta$ remains unchanged by reordering the columns. It follows that if $\alpha_{\mathfrak{i}}^{\prime}$ denotes a $1 \times v$ row vector each elenent of which is $t_{i}$, the matrix

$$
\left[\begin{array}{ll}
\alpha_{v}^{\prime}, & \alpha_{1}^{\prime}, \cdots, \alpha_{v-1}^{\prime} \\
\Delta_{0}, & \Delta^{(1)}, \cdots, \Delta^{(v-1)}
\end{array}\right]
$$


is an orthogonal array $\left[v^{2}, q+1, v, 2\right]$ of order $v^{2}, q+1$ constraints, $v$ levels and strength 2 , and can therefore be used to construct a set of $q-1$ m.o.l.s. of order $v$.

5. Use of balanced incomplete block designs for obtaining sets of mutually orthogonal Latin squares. A balanced incomplete block (BIB) design with parameters $v, b, r, k, \lambda$ is an arrangement of $v$ objects or treatments into $b$ sets or blocks such that (i) each block contains $k<v$ different treatments, (ii) each treatment occurs in $r$ different blocks, (iii) each pair of treatments occurs together in exactly $\lambda$ blocks. The parameters satisfy the relations

$$
\lambda(v-1)=r(k-1), \quad b k=v r, \quad b \geqq v .
$$

These conditions are necessary but not sufficient for the existence of a BIB design. BIB designs were first introduced into statistical studies by Yates [21], but occur in earlier literature in connection with various combinatorial problems. Subsequent to Yates many authors have dealt with the problem of constructing these designs. Without attempting a complete bibliography we shall only refer to [2]. A BIB design is said to be symmetrical if $v=b$, and in consequence $k=r$. A BIB design is said to be resolvable [3] if the blocks can be divided into sets, such that the blocks of any given set contain each treatment exactly once.

A BIB design with $\lambda=1$ is clearly a pairwise balanced design of index unity and type $(v ; k)$. We shall denote such a design by $\mathrm{BIB}(v ; k)$. It is evident that a symmetric or a resolvable BIB design is separable, since in the first case the blocks constitute a single set of type $\mathrm{I}$, and in the second case the blocks can be divided into $r$ subsets of type II. From Theorem 1 we have

THEOREM 2. If there exists a BIB design with $v$ treatments, block size $k$ and $\lambda=1$, then $N(v) \geqq N(k)-1$. If the BIB design is separable $N(v) \geqq N(k)$.

When $k$ is a prime power $N(k)=k-1$, hence we have Parker's theorem [18] mentioned in the introduction which asserts that $N(v) \geqq k-2$, when $k$ is a prime power. This result can be improved by unity when the design is separable.

THEOREM 3. $N(v) \geqq N(k)$ in the following cases:

(i) $v=s^{2}+s+1, k=s+1$, where $s$ is a prime power,

(ii) $v=s^{3}+1, k=s+1$, where $s$ is a prime power,

(iii) $v=s(s-1) / 2, k=s / 2=2^{m-1}, m \geqq 2$.

Proof. Part (i) follows from Theorem 2, since there exists a symmetrical BIB design with parameters $v=b=s^{2}+s+1, r=k=s+1, \lambda=1$ when $s$ is a prime power [2, p. 364]. The existence of the design is equivalent to the existence of a projective plane $\mathrm{PG}(2, s)$ of order $s$.

Example (1). If $s=4, N(k)=N(5)=4$. Hence there exist at least 4 m.o.l.s. of order 21. The MacNeish-Mann result only assures the existence of 2 orthogonal Latin squares. 
EXAmple (2). If $s$ is a Mersenne prime $>3$ then $k=s+1=2^{p}$ and $N(k)$ $=2^{p}-1$. Hence $N\left(s^{2}+s+1\right) \geqq s$, whereas $n\left(s^{2}+s+1\right)=2$, e.g., if we take $s=31$ then $N(993) \geqq 31, n(993)=2$.

ExAmple (3). If $s=49, k=50$. Parker's theorem is inapplicable. It is shown in $\S 6$, Example (7), that $N(50) \geqq 5$. Hence $N\left(49^{2}+49+1\right) \geqq 5$ whereas $n\left(49^{2}+49+1\right)=2$.

Part (ii) follows from the existence of the series of resolvable BIB designs

$$
v=s^{3}+1, \quad b=s^{2}\left(s^{2}-s+1\right), \quad r=s^{2}, \quad k=s+1, \quad \lambda=1,
$$

which has been proved in [6].

EXAmple (4). Let $v=31^{3}+1=2^{5} \cdot 7^{2} \cdot 19=29792, k=32=2^{5}$. Hence $N(v)$ $\geqq 31$ whereas $n(v)=18$.

To prove part (iii) we shall establish the existence of a series of resolvable BIB designs with parameters

$$
v=s(s-1) / 2, \quad b=s^{2}-1, \quad r=s+1, \quad k=s / 2=2^{m-1}, \quad \lambda=1 .
$$

Consider the finite projective plane $\operatorname{PG}\left(2,2^{m}\right)$, and take a nondegenerate conic $C$ on this plane, e.g., the conic

$$
x z=y^{2} .
$$

We know $\left[4\right.$, p. 158] that there are $s+1$ points $P_{1}, P_{2}, \cdots, P_{s+1}$ on this conic, where $s=2^{m}$. Through any point $P_{i}=\left(x^{\prime}, y^{\prime}, z^{\prime}\right)$ on the conic there will pass $s+1$ lines $s$ of which meet the conic in the other $s$ points on the conic, and the remaining line $z^{\prime} x+x^{\prime} z=0$, meets the conic in the single point $P_{i}$. This may be called a tangent to the conic at $P_{i}$. The $s+1$ tangents to the conic all pass through the point $P_{0}=(0,1,0)$ which may be called the pole of the conic. The $s^{2}+s+1$ lines of the plane may be divided into three classes: (a) The $s(s+1) / 2$ intersectors each of which meets the conic in 2 points but does not pass through $P_{0}$; (b) The $s+1$ tangents each of which meets the conic in one point and passes through $P_{0}$; (c) The $s(s-1) / 2$ nonintersectors which do not meet the conic and hence do not pass through $P_{0}$. Let the $s^{2}-1$ points of the plane other than $P_{0}$ and the points of the conic be called retained points. Consider the configuration of the retained points and nonintersectors. If the retained points are called blocks, and the nonintersectors are called treatments, and if treatment is considered to belong to a block if the corresponding line and point are incident then we get the design (5.1). This follows from the fact that on each nonintersector there lie $s+1$ points. This gives $r=s+1$. Also through each retained point $P$ there passes one tangent, viz. $P P_{0}$. If the point of contact of this tangent is $P_{i}$ then the other $s$ points on the conic lie two by two on $s / 2$ intersectors through $P$. Hence there pass $s / 2$ nonintersectors through $P$. This gives $k=s / 2$. That $\lambda=1$ follows from the fact that any two nonintersectors meet in one and only one point which is a retained point.

Next we have to prove that the design is resolvable, i.e., the blocks 
can be separated in groups. We have to find sets of $s-1$ blocks which contain each treatment exactly once, i.e., sets of $s-1$ retained points such that each nonintersector passes through one and only one point of the set. The $s-1$ retained points on any intersector or tangent provide such a set. Finally we have to choose a set of $s+1$ intersectors or tangents which contain between them all the retained points. Such a set is provided by the $s+1$ tangents, or the $s+1$ lines through any point on the conic. This proves the resolvability of the design.

Example (5). Parker leaves undecided the question whether there are an infinity of values of $v$ for which $N(v)>n(v)$. This question is decided in the affirmative by part (iii) of our theorem, since if we take $m=2 n$, then $N(v)$ $\geqq N\left(2^{m-1}\right)=2^{2 n-1}-1$. Also $v=2^{2 n-1}\left(2^{n}+1\right)\left(2^{n}-1\right)$. Hence $n(v) \leqq 2^{n}-2<N(v)$. For example, let $m=4$, then $v=120, \quad k=8, \quad N(120) \geqq N(k)=7, \quad n(120)$ $=n(8 \cdot 5 \cdot 3)=2$.

If from a BIB design with $v$ treatments, block size $k$ and $\lambda=1$, we omit $x \leqq k$ treatments belonging to any block, we obviously get a pairwise balanced design of index unity and type $(v-x ; k, k-1, k-x)$. Applying Theorem 1 , we get

THEOREM 4. Existence of a BIB design with $v$ treatments, block size $k$ and $\lambda=1$, implies that

$$
N(v-x) \geqq \min (N(k), N(k-1), N(k-x))-1
$$

if $x \leqq k$.

EXAmple (6). Consider the symmetrical BIB design $v=b=s^{2}+s+1$, $r=k=s+1, \lambda=1$, when $s=16$. If we take $x=6,8$ and 9 respectively, we obtain $N(267) \geqq 9, \quad N(265) \geqq 7, \quad N(264) \geqq 6$, whereas $n(267)=2, \quad n(265)=4$, $n(264)=2$.

6. The falsity of Euler's conjecture regarding the nonexistence of two orthogonal Latin squares of order $v \equiv 2(\bmod 4)$, and the method of adjunction of treatments.

LEMma 3. There exists a resolvable series of BIB designs with parameters (6.1) $v^{*}=24 m+15, b^{*}=(8 m+5)(12 m+7), r^{*}=12 m+7, k^{*}=3, \lambda^{*}=1$.

Proof. The existence of the design is equivalent to a solution of the Kirkman school girl problem for triplets with $n=24 m+15$ girls. There is an extensive literature on this problem and a complete bibliography of all work up to 1911 is given in [12], and an excellent summary of the known results is contained in [1, Chapter X, pp. 267-298]. The problem is solvable for all values of $n$ of the form $6 t+3$. The special case required by us is $t=4 m+2$. The girls can be considered as the treatments of (6.1), triplets as blocks, and each day's walk as a complete replication. 
In the special case when $12 m+7$ is a prime power, an analytical solution can be given as follows. We first prove

Lemma 4. If $x$ is a primitive element of the Galois field $\mathrm{GF}(12 m+7)$, where $12 m+7$ is a prime power, then there exist odd integers $q$ and $\alpha$ such that

$$
x^{q}=\left(x^{\alpha}+1\right) /\left(x^{\alpha}-1\right) .
$$

Proof. The lemma is proved by considering the $(1,1)$ correspondence

$$
y=(z+1) /(z-1)
$$

between the elements of GF $(12 m+7)$, augmented by infinity. The correspondence is an involution and it is easily shown that there must exist a pair of corresponding odd powers of $x$.

To each element $u$ of the field GF $(12 m+7)$ let there correspond two treatments $u_{1}$ and $u_{2}$. To the $24 m+14$ treatments thus obtained adjoin the treatment $\infty$. The existence and resolvability of the design can now be proved by using the second fundamental theorem of the method of differences developed in [2]. Consider the set of $8 m+5$ initial blocks

$$
\begin{aligned}
& \left(x_{1}^{2 i}, x_{1}^{2 i+4 m+2}, x_{1}^{2 i+8 m+4}\right), \quad i=0,1, \cdots, 2 m \text {; } \\
& \left(x_{1}^{\alpha+j}, x_{2}^{2 j}, \quad x_{2}^{6 m+3+2 j}\right), \quad j=0,1, \cdots, 6 m+2 \text {; } \\
& \left(0_{1}, 0_{2}, \infty\right) \text {. }
\end{aligned}
$$

Using methods on pages 374 and 384 of [2] it is easy to verify that all the nonzero pure differences and all the mixed differences are symmetrically repeated once. Hence the design (6.1) can be obtained by developing the initial blocks. Since the set of initial blocks gives a complete replication, the design is resolvable.

We can now proceed to prove the main theorem of this section.

THEOREM 5. There exist at least two orthognal Latin squares of order $v=36 m+22$, where $m$ is any non-negative integer.

Proof. Let us start with the BIB design whose parameters are given by (6.1). Take $12 m+7$ new treatments $\theta_{1}, \theta_{2}, \cdots, \theta_{12 m+7}$ and to each block of the $i$ th replication add the treatment $\theta_{i}$. Also take a new block consisting of the new treatments. The augmented design is now a pairwise balanced design of index unity of the type $\left(v ; k_{1}, k_{2}\right)$ where $v=36 m+22, k_{1}=4, k_{2}=12 m+7$. Since $q_{1}-1=N\left(k_{1}\right)=3, q_{2}-1=N\left(k_{2}\right) \geqq n(12 m+7) \geqq 4, q=\min \left(q_{1}, q_{2}\right)=4$, it follows from Theorem 1 , that there exist at least two orthogonal Latin squares of order $v=36 m+22$.

Two superposed orthogonal Latin squares of order 22 constructed by this method are exhibited in the Appendix, the capital letters corresponding to the first square and the small letters to the second. 
We shall now show that there exist numbers $v \equiv 2(\bmod 4)$ for which there exist many more than two mutually orthogonal Latin squares of order $v$.

THEOREM 6. If $s$ and $s+1$ are both prime powers, then for any integer $m \geqq 2$.

$$
N\left(s^{m}+1\right) \geqq s-2 .
$$

Proof. Consider the Euclidean geometry $\operatorname{EG}(m, s)$. Taking the points and lines of this geometry as the treatments and blocks respectively, we get a BIB design with parameters $v^{*}=s^{m}, b^{*}=r^{*} s^{m-1}, r^{*}=1+s+\cdots+s^{m-1}$, $k^{*}=s, \lambda^{*}=1$. Further the design is resolvable into $r^{*}$ replications corresponding to the lines of the Euclidean geometry passing through a point of the $(m-1)$-flat at infinity of the projective geometry $\operatorname{PG}(m, s)$ in which the Euclidean geometry can be embedded. Add a new treatment $\theta$ to the blocks of the first replication. This is equivalent to the adjunction of a point at infinity to the lines of a parallel pencil. The augmented design is a pairwise balanced design of index unity and type $\left(s^{m}+1 ; s+1, s\right)$. Since $s+1$ and $s$ are both prime powers Theorem 1 gives $N\left(s^{m}+1\right) \geqq s-2$.

We may note that $s$ and $s+1$ will be prime powers if (i) $s$ is a Mersenne number, (ii) $s+1$ is a Fermat prime, (iii) $s=8$.

REMARK. If $s$ is a Mersenne number, $s^{2 l}+1 \equiv 2(\bmod 4)$. Hence if $s>3$, this provides another infinite set of values $v$ of the form $4 t+2$ for which at least $s-2$ m.o.l.s. of order $v$ can be constructed, thus again disproving Euler's conjecture.

EXAMPLE (7). Taking $l=1$ and $s=7,8$ or 31 , we get $N(50) \geqq 5, N(65) \geqq 6$, $N(962) \geqq 29$.

Instead of one we may add $x$ new treatments, $x \leqq r^{*}$, one to each block of a complete replication, and add a new block containing the new treatments. We get a pairwise balanced design of index unity and type $\left(s^{m}+x ; s+1, s, x\right)$. Hence the following:

Corollary 1. If $s$ and $s+1$ are prime powers and $1<x \leqq 1+s+\cdots$ $+s^{m-1}, m \geqq 2, N\left(s^{m}+x\right) \geqq \min (s-1, N(x))-1$.

ExAmple (8). Taking $s=7, m=2, x=5$, we get $N(54) \geqq 3$.

If we do not insist that $s$ and $s+1$ are both prime powers in the above theorem, then we get

CoRollary 2. If $s$ is a prime power, and $m \geqq 2$, then

$$
N\left(s^{m}+1\right) \geqq \min (s-1, N(s+1))-1,
$$

and if $1<x \leqq 1+s+\cdots+s^{m-1}$

$$
N\left(s^{m}+x\right) \geqq \min (s-1, N(s+1), N(x))-1 .
$$

EXAmple (9). Let $s=19, m=2$. Then $N(s+1)=N(20) \geqq n(20)=3$. Hence $N(362) \geqq 2$. 
THEOREM 7. If there exist at least two orthogonal Latin squares of order $4 t+2$, then there exist at least two orthogonal Latin squares of any order $v$ which is an odd multiple of $4 t+2$.

Proof. Let $v_{1}=4 t+2, v_{2}=2 u+1$ and $v=v_{1} v_{2}$. Then from Lemma 1

$$
N(v) \geqq \min \left(N\left(v_{1}\right), N\left(v_{2}\right)\right) \text {. }
$$

Now $N\left(v_{1}\right) \geqq 2$ by hypothesis, and since $v_{2}$ is odd $N\left(v_{2}\right) \geqq n\left(v_{2}\right) \geqq 2$. This proves the theorem.

Example (10). Let $v=110=22 \cdot 5$. From Theorem $5, N(22) \geqq 2$. Hence $N(110) \geqq 2$.

The method of adjunction can obviously be applied to any resolvable $\mathrm{BIB}$ designs. In particular taking the design given by parameters (5.1), we get

TheOREM 8. If $s=2^{m}, m \geqq 3$, then

$$
N\left(\frac{s(s-1)}{2}+1\right) \geqq \min \left(\frac{s}{2}-1, N\left(\frac{s}{2}+1\right)\right)-1,
$$

and if $1<x \leqq s+1$,

$$
N\left(\frac{s(s-1)}{2}+x\right) \geqq \min \left(\frac{s}{2}-1, N\left(\frac{s}{2}+1\right), N(x)\right)-1 .
$$

EXAMPLE (11). Taking $s=16, x=9$, we get $N(129) \geqq 6$, whereas $n(129)=2$.

7. Certain pairwise balanced designs of index unity associated with the finite planes $\operatorname{PG}\left(2,2^{3}\right)$ and $\operatorname{EG}\left(2,3^{2}\right)$, and the corresponding sets of mutually orthogonal Latin squares. We shall now consider certain designs based on finite geometry which take advantage of the fact that 7,8 and 9 are three consecutive integers which are prime powers.

Let us consider the finite projective plane $\mathrm{PG}\left(2,2^{3}\right)$. It has been shown in proving part (iii) of Theorem 3 , that there exists on this plane a set $\Omega$ of 10 points, viz., the set of points $P_{1}, P_{2}, \cdots, P_{9}$ on a nondegenerate conic $C$, and its pole $P_{0}$, such that every line on the plane intersects $\Omega$ in either 2 points or no points. Let $\Omega^{*}$ be a subset of $\Omega$. Then the number of points in $\Omega^{*}$ is $x \leqq 10$. Consider the set of retained points obtained by deleting from the plane the points of $\Omega^{*}$. If we take the set of retained points as treatments, and lines as blocks, then clearly each block contains either 7,8 or 9 points. Also any pair of retained points occurs exactly in one block. Hence we obtain a pairwise balanced design of index unity and type $(73-x ; 7,8,9)$. Applying Theorem 1, we have

Theorem 9. $N(73-x) \geqq 5$ if $x \leqq 10$.

Example (12). Taking $x=3$ or $x=7$ we find that there exist at least 5 m.o.l.s. of orders 70 or 66 . 
Again consider the Euclidean plane $\operatorname{EG}\left(2,3^{2}\right)$. If $a x^{2}+2 h x y+b y^{2}$ is irreducible in $\mathrm{GF}\left(3^{2}\right)$, where $a, b, c$ belong to this field, and if $f, g, h$ are so chosen that

$$
\Delta=a b c+2 f g h-a f^{2}-b g^{2}-c h^{2} \neq 0
$$

then the conic $a x^{2}+2 h x y+b y^{2}+2 g x+2 f y+c=0$ is nondegenerate and does not meet the line at infinity. If $\Omega$ is the set of 10 points on this conic, then no line meets $\Omega$ in more than two points. If we take $\Omega^{*}$ to be a subset of $\Omega$, then the set of retained points obtained by deleting the points of $\Omega^{*}$ from the plane, provide a pairwise balanced design of the type $(81-x ; 7,8,9)$ where $x \leqq 10$. Hence using Theorem 1 we have

Theorem 10. $N(81-x) \geqq 5$, if $x \leqq 10$.

EXAmple (13). Taking $x=3$ or 7 we find that there exist at least 5 m.o.l.s. of orders 78 and 74.

8. Use of group divisible designs in constructing sets of mutually orthogonal Latin squares. An arrangement of $v$ objects (treatments) in $b$ sets (blocks) each containing $k$ distinct treatments is said to be a group divisible (GD) design if the treatments can be divided into $l$ groups of $m$ treatments each, so that any two treatments belonging to the same group occur together in $\lambda_{1}$ blocks, and any two treatments from different groups occur together in $\lambda_{2}$ blocks. We will denote such a design by the notation $\operatorname{GD}\left(v ; k, m ; \lambda_{1}, \lambda_{2}\right)$. The combinatorial properties of these designs have been studied in [7] where it has been shown that

$$
v=l m, \quad b k=v r, \quad \lambda_{1}(m-1)+\lambda_{2} m(l-1)=r(k-1),
$$

$r$ being the number of replications, i.e., the number of times each treatment occurs in the design. It has also been shown that

$$
P=r k-\lambda_{2} v \geqq 0, \quad Q=r-\lambda_{1} \geqq 0 .
$$

The GD designs can be divided into three classes.

(i) Regular (R) characterized by $P>0, Q>0$.

(ii) Semiregular (SR) characterized by $P=0, Q>0$.

(iii) Singular (S) characterized by $Q=0$.

Methods of constructing these designs have been given in [8]. So far as the construction of m.o.l.s. is concerned a special role is played by GD designs with $\lambda_{1}=0, \lambda_{2}=1$, which in our notation can be denoted by $\mathrm{GD}(v ; k, m ; 0,1)$. If further this design is regular we shall denote it by $\operatorname{RGD}(v ; k, m ; 0,1)$ and if it is semiregular we shall denote it by $\operatorname{SRGD}(v ; k, m ; 0,1)$.

Lemma 5. Existence of a $\mathrm{GD}(v ; k, m ; 0,1)$ implies the existence of pairwise balanced design $(D)$ of index unity and type $(v ; k, m)$.

If to the $b$ blocks of the GD design, we add $l$ new blocks corresponding to 
the groups we get a pairwise balanced design of index unity and type $(v ; k, m)$. It follows from Theorem 1 :

Theorem 11. If there exists a $\mathrm{GD}(v ; k, m ; 0,1)$, then $N(v) \geqq \min (N(k), N(m))$ -1 . Further if the GD design is separable $N(v) \geqq \min (N(k), N(m))$.

Corollary. If $s$ is a prime power $N\left(s^{2}-1\right) \geqq N(s-1)$.

Proof. Since $s$ is a prime power $N(s)=s-1$, and we know from $[8$, p. 173] that there exists a symmetric and hence separable $\operatorname{RGD}\left(s^{2}-1 ; s, s-1 ; 0,1\right)$.

EXAmpLE (14). Taking $s=9, N(80) \geqq N(8)=7$, whereas $n(80)=4$. We note that Parker's result is inapplicable in this case since there does not exist a BIB design with $v=80$, and $k>2, \lambda=1$. Taking $s=32$, we get $N(1023)$ $\geqq N(31)=30$ whereas $n(1023)=2$. where

Theorem 12. Suppose there exists $a \mathrm{GD}(v ; k, m ; 0,1)$. Then $N(v-1) \geqq q-2$

$$
q-1=\min (N(k), N(k-1), N(m), N(m-1)) .
$$

If further the GD design is resolvable then $N(v-1) \geqq q-1$.

Proof. From Lemma 5, we get a pairwise balanced design $(D)$ of index unity and type $(v ; k, m)$. Omitting a particular treatment say $\alpha$ from the blocks in which it occurs, we get a pairwise balanced design $\left(D_{\alpha}\right)$ of the type $(v-1 ; k, k-1, m, m-1)$. Theorem 1 now gives $N(v-1) \geqq q-2$.

We shall now show that we can get $q-1$ m.o.l.s. when the GD design is resolvable. There are $l$ blocks in $(D)$, each of which contains $m$ treatments, viz., the blocks corresponding to the groups. Consider the corresponding set of blocks of $\left(D_{\alpha}\right)$. Since only one group contains the treatment $\alpha$, this set of blocks consists of one block $\delta_{g, m-1}$ containing $m-1$ treatments, and a set $\left(S_{g, m}\right)$ of $l-1$ blocks each of which contains $m$ treatments. Since the initial GD design is resolvable, the other blocks of $(D)$ are divisible into $r$ sets, such that each set of blocks contains every treatment exactly once. From the $i$ th of these sets, we obtain by dropping $\alpha$ the corresponding set of blocks of $\left(D_{\alpha}\right), i=1,2, \cdots, r$. This set consists of $v / k$ blocks, one of which $\delta_{i, k-1}$ contains $k-1$ treatments and a set $\left(S_{i, k}\right)$ of $(v / k)-1$ blocks each of which contains $k$ treatments.

Put $q_{3}=N(m-1)+1$ and $q_{4}=N(m)+1$. Let $P_{3}$ and $P_{3 c}, c=1,2, \cdots$, $m-2$ be the matrices of order $q_{3} \times(m-1)(m-2)$ and $q_{3} \times(m-1)$ respectively of Lemma 2. Similarly let $P_{4}$ and $P_{4 d}, d=1,2, \cdots, m-1$ be matrices of order $q_{4} \times m(m-1)$ and $q_{4} \times m$ respectively.

Let $\Delta_{g}$ be the matrix of order $q \times((m-1)(m-2)+m(m-1)(l-1))$ obtained from

$$
\left[P_{3}\left(\delta_{g, m-1}\right), P_{4}\left(S_{g, m}\right)\right]
$$

by retaining the first $q$ rows. Defining column equivalence as in the proof of Theorem 1 and remembering that (i) the number of submatrices $P_{4 d}$ is one 
greater than the number of submatrices $P_{3 c}$, (ii) the block $\delta_{g, m-1}$ and the set of blocks $\left(S_{g, m}\right)$ contain between them all the $v-1$ treatments in the design $\left(D_{\alpha}\right)$ we can write

$$
\Delta_{g} \sim\left[\Delta_{g}^{(1)}, \Delta_{g}^{(2)}, \cdots, \Delta_{g}^{(m-2)}, \Delta_{g}^{*}\right],
$$

where each row of $\Delta_{g}^{(j)}$ contains each of the $v-1$ retained treatments exactly once $(j=1,2, \cdots, m-2)$. Thus $\Delta_{o}^{(j)}$ possesses the property $\tau_{1}$ relative to the $v-1$ retained treatments. Each row of $\Delta_{o}^{*}$ contains all the $v-m$ retained treatments other than those contained in $\delta_{g, m-1}$. Also any pair of treatments occurring in the block $\delta_{g, m-1}$ or blocks $\left(S_{g, m}\right)$ of $\left(D_{\alpha}\right)$ occurs as a column exactly once in any two rowed submatrix of $\Delta_{g}$.

Likewise from $\delta_{i, k-1}$ and $\left(S_{i, k}\right)$ we get a $q \times((k-1)(k-2)+(k-1)(v-k))$ matrix $\Delta_{i}$

$$
\Delta_{i} \sim\left[\Delta_{i}^{(1)}, \Delta_{i}^{(2)}, \cdots, \Delta_{i}^{(k-2)}, \Delta_{i}^{*}\right], \quad i=1,2, \cdots, r,
$$

possessing similar properties in relation to the blocks $\delta_{i, k-1}$ and $\left(S_{i, k}\right)$.

Let $\Delta_{0}$ be a $q \times(v-1)$ matrix whose $j$ th column contains the $j$ th treatment in $\left(D_{\alpha}\right)$ in every position. It follows from the property of pairwise balance possessed by $(D)$, that the set of blocks $\delta_{g, m-1}, \delta_{1, k-1}, \cdots, \delta_{r, k-1}$ taken together contain each of the $v-1$ retained treatments exactly once. Let $\Delta_{g}^{* *}$ be a $q \times(m-1)$ matrix each of whose rows is $\delta_{g, m-1}^{\prime}$. Similarly let $\Delta_{i}^{* *}$ be a $q \times(k-1)$ matrix each of whose rows is $\delta_{i, k-1}^{\prime}(i=1,2, \cdots, r)$. Then

$$
\Delta_{0} \sim\left[\Delta_{o}^{* *}, \Delta_{1}^{* *}, \cdots, \Delta_{r}^{* *}\right]
$$

clearly

$$
\Delta=\left[\Delta_{g}, \Delta_{1}, \cdots, \Delta_{r}\right],
$$

has the property $\tau_{2}$ in relation to the $v-1$ treatments of $\left(D_{\alpha}\right)$. It follows from this or can be directly verified using (8.1) that $\Delta$ is of order $q \times(v-1)(v-2)$. Hence $\left[\Delta_{0}, \Delta\right]$ is an orthogonal array $\left[(v-1)^{2}, q, v-1,2\right]$ of order $(v-1)^{2}, q$ constraints, $v-1$ levels and strength 2 .

We now note that the matrices $\left[\Delta_{0}^{*}, \Delta_{0}^{* *}\right]$ and $\left[\Delta_{\imath}^{*}, \Delta_{i}^{* *}\right], i=1,2, \cdots, r$ have the property $\tau_{1}$, that each row contains every treatment of $\left(D_{\alpha}\right)$ exactly once. Hence by a reordering of columns, $\left[\Delta_{0}, \Delta\right]$ can be divided into $(v-1)$ submatrices of order $q \times(v-1)$ such that each submatrix has the property $\tau_{1}$. After this reordering has been made $\left[\Delta_{0}, \Delta\right]$ can be converted into an orthogonal array $\left[(v-1)^{2}, q+1, v-1,2\right]$ of $q+1$ constraints, by adding another row such that the part of this row corresponding to the $c$ th submatrix with the property $\tau_{1}$ consists of the $c$ th treatment repeated $v-1$ times. It follows that there exist $q-1$ m.o.l.s. of order $v-1$. This proves the second part of Theorem 12 .

In the same manner we can prove: 
COROLlaRy. If there exists a resolvable BIB $(v ; k)$ then

$$
N(v-1) \geqq \min (N(k), N(k-1)) .
$$

Let there exist a $\operatorname{GD}(v ; k, m ; 0,1)$. Then by taking a new treatment $\theta$, and adding it to each of the $l$ blocks of the design $(D)$ of Lemma 5 , we get a pairwise balanced design of index unity and type $(v+1 ; k, m+1)$. Using Theorem 1, we have

Theorem 13. If there exists $a \mathrm{GD}(v ; k, m ; 0,1)$ then

$$
N(v+1) \geqq \min (N(k), N(m+1))-1 \text {. }
$$

Suppose the design $\mathrm{GD}(v ; k, m ; 0,1)$ is resolvable and $r$ is the number of replications. We can take new treatments $\theta_{1}, \theta_{2}, \cdots, \theta_{x}, x \leqq r$, and add $\theta_{i}$ to each block of the $i$ th replication. Also we take blocks corresponding to the groups of treatments and a new block consisting of the treatments $\theta_{1}, \theta_{2}, \cdots, \theta_{x}$ when $x>1$. We then get a pairwise balanced design of index unity and type $(v+x ; k+1, k, m)$ if $x=1$, type $(v+x ; k+1, k, m, x)$ if $1<x<r$ and type $(v+x ; k+1, m, x)$ if $x=r$. Using Theorem 1 , we get

Theorem 14. Suppose there exists a resolvable $\mathrm{GD}(v ; k, m ; 0,1)$, then $N(v+x) \geqq \min (N(k+1), N(k), N(m))-1$ if $x=1, N(v+x) \geqq \min (N(k+1)$, $N(k), N(m), N(x))-1$ if $1<x<r$ and $N(v+x) \geqq \min (N(k+1), N(m), N(x))$ -1 , if $x=r$.

Theorems 12, 13 and 14 can be applied to semiregular GD designs, which provide a large class of resolvable GD designs. The following result is taken from $[8$, p. 181].

Lemma 6. The existence of an $\operatorname{SRGD}(l m ; l, m ; 0,1)$ implies the existence of an orthogonal array $A=\left(m^{2}, l, m, 2\right)$ of strength 2 (or equivalently the existence of a set of $l-2$ m.o.l.s. of order $m)$, and conversely.

Suppose there exists an $\operatorname{SRGD}(l m ; l, m ; 0,1)$. Then from Lemma 6 , there exists an orthogonal array $A=\left(m^{2}, l, m, 2\right)$. Without loss of generality we can take the first row of $A$ as $\left(\epsilon_{1}^{\prime}, \epsilon_{2}^{\prime}, \cdots, \epsilon_{m}^{\prime}\right)$ where $\epsilon_{i}^{\prime}$ is a $1 \times m$ row vector each of whose elements is $i$. If we now delete the first row, there remains an orthogonal array $A_{1}=\left(m^{2}, l-1, m, 2\right)$ of $l-1$ constraints which can be subdivided into $m$ submatrices each of order $(l-1) \times m$ possessing the property $\tau_{1}$ that every row of each submatrix contains the symbols $1,2, \cdots, m$ exactly once. This property remains unchanged if we get $A_{2}=\left(m^{2}, l_{1}, m, 2\right)$ by deleting some rows of $A_{1}, l_{1} \leqq l-1$. Replace the symbol $j$ in the $i$ th row of $A_{2}$ by the treatment $\theta_{i j}\left(i=1,2, \cdots, l_{1} ; j=1,2, \cdots, m\right)$, and take the columns as blocks. We then get an $\operatorname{SRGD}\left(l_{1} m ; l_{1}, m ; 0,1\right)$ for which the groups are given by the $m$ treatments occurring in any given row. This design is resolvable, the blocks corresponding to the submatrices with the prop- 
TABLE I

\begin{tabular}{|c|c|c|c|}
\hline$v$ & $n(v)$ & 1.b. for $N(v)$ & Remarks \\
\hline 21 & 2 & 4 & Th. 3, Ex. (1) \\
\hline $22^{*}$ & 1 & 2 & Th. $5, m=0$ \\
\hline 24 & 2 & 3 & Th. 11 , Cor. $s=5$ \\
\hline 39 & 2 & 3 & Th. 12, Ex. (15) \\
\hline $50^{*}$ & 1 & 5 & Th. 6, Ex. (7) \\
\hline $54^{*}$ & 1 & 3 & Th. 6, Ex. (8) \\
\hline 57 & 2 & 7 & Th. 2 , BIB $(57 ; 8)$ \\
\hline $58^{*}$ & 1 & 2 & Th. $5, m=1$ \\
\hline 65 & 4 & 6 & Th. 6, Ex. (7) \\
\hline $66^{*}$ & 1 & 5 & Th. $9, x=7$ \\
\hline 68 & 3 & 5 & Th. $9, x=5$ \\
\hline 69 & 2 & 5 & Th. $9, x=4$ \\
\hline $70^{*}$ & 1 & 5 & Th. $9, x=3$ \\
\hline $74^{*}$ & 1 & 5 & Th. $10, x=7$ \\
\hline 75 & 2 & 5 & Th. $10, x=6$ \\
\hline 76 & 3 & 5 & Th. $10, x=5$ \\
\hline $78^{*}$ & 1 & 5 & Th. $10, x=3$ \\
\hline 80 & 4 & 7 & Th. 11 , Cor. $s=9$ \\
\hline $82^{*}$ & 1 & 3 & Th. 14, Ex. (17) \\
\hline 84 & 2 & 5 & $\operatorname{SRGD}(77 ; 7,11 ; 0,1)$, Th. $14, x=7$ \\
\hline 85 & 4 & 5 & SRGD $(77 ; 7,11 ; 0,1)$, Th. $14, x=8$ \\
\hline $86^{*}$ & 1 & 5 & Th. 14, Ex. (17) \\
\hline 92 & 3 & 5 & $\operatorname{SRGD}(91 ; 7,13 ; 0,1)$, Th. $14, x=1$ \\
\hline $94^{*}$ & 1 & 2 & Th. $5, m=2$ \\
\hline 95 & 4 & 5 & SRGD $(88 ; 8,11 ; 0,1)$, Th. $14, x=7$ \\
\hline 96 & 2 & 6 & SRGD $(88 ; 8,11 ; 0,1)$, Th. $14, x=8$ \\
\hline $98^{*}$ & 1 & 5 & SRGD $(91 ; 7,13 ; 0,1)$, Th. $14, x=7$ \\
\hline 100 & 3 & 5 & SRGD $(91 ; 7,13 ; 0,1)$, Th. $14, x=9$ \\
\hline $102^{*}$ & 1 & 5 & SRGD $(91 ; 7,13 ; 0,1)$, Th. $14, x=11$ \\
\hline 105 & 2 & 6 & SRGD $(104 ; 8,13 ; 0,1)$, Th. $14, x=1$ \\
\hline $110^{*}$ & 1 & 2 & Th. 7, Ex. (10) \\
\hline 111 & 2 & 5 & SRGD $(104 ; 8,13 ; 0,1)$, Th. $14, x=7$ \\
\hline 115 & 4 & 6 & SRGD $(104 ; 8,13 ; 0,1)$, Th. $14, x=11$ \\
\hline 120 & 2 & 7 & Th. 3 , pt. (iii), $s=16$ \\
\hline 123 & 2 & 5 & SRGD $(112 ; 7,16 ; 0,1)$, Th. $14, x=11$ \\
\hline $126^{*}$ & 1 & 5 & SRGD $(119 ; 7,17 ; 0,1)$, Th. $14, x=7$ \\
\hline 129 & 2 & 6 & Th. 8, Ex. (11) \\
\hline $130^{*}$ & 1 & 5 & SRGD $(119 ; 7,17 ; 0,1)$, Th. $14, x=11$ \\
\hline 132 & 2 & 5 & SRGD $(119 ; 7,17 ; 0,1)$, Th. $14, x=13$ \\
\hline $134^{*}$ & 1 & 5 & SRGD $(133 ; 7,19 ; 0,1)$, Th. $14, x=1$ \\
\hline 135 & 4 & 6 & Th. 12, Ex. (15) \\
\hline $138^{*}$ & 1 & 3 & SRGD $(133 ; 7,19 ; 0,1)$, Th. $14, x=5$ \\
\hline 140 & 3 & 5 & SRGD $(133 ; 7,19 ; 0,1)$, Th. $14, x=7$ \\
\hline 141 & 2 & 6 & SRGD $(128 ; 8,16 ; 0,1)$, Th. $14, x=13$ \\
\hline $142^{*}$ & 1 & 5 & SRGD $(133 ; 7,19 ; 0,1)$, Th. $14, x=9$ \\
\hline 145 & 4 & 7 & Th. 13, Ex. (16) \\
\hline $146^{*}$ & 1 & 5 & SRGD $(133 ; 7,19 ; 0,1)$, Th. $14, x=13$ \\
\hline 147 & 2 & 6 & SRGD $(136 ; 8,17 ; 0,1)$, Th. $14, x=11$ \\
\hline $150^{*}$ & 1 & 5 & SRGD $(133 ; 7,19 ; 0,1)$, Th. $14, x=17$ \\
\hline
\end{tabular}


erty $\tau_{1}$ give a complete replication. Clearly $r=m$ for this design. We thus have

LEMMA 7. The existence of an $\operatorname{SRGD}(l m ; l, m ; 0,1)$ implies the existence of a resolvable $\operatorname{SRGD}\left(l_{1} m ; l_{1}, m ; 0,1\right)$, and an orthogonal array $A_{2}=\left(m^{2}, l_{1}, m, 2\right)$ divisible into submatrices with property $\tau_{1}$, for any $l_{1}<l$.

CoRollaRy. There exists a resolvable $\operatorname{SRGD}\left(l_{1} m ; l_{1}, m ; 0,1\right)$ if $l_{1} \leqq N(m)+1$.

Proof. Let $l=N(m)+2$. Then there exists an orthogonal array $\left(m^{2}, l, m, 2\right)$. From Lemma 6 , there exists an $\operatorname{SRGD}(l m ; l, m ; 0,1)$. The required result now follows from Lemma 7.

Example (15). From $\operatorname{SRGD}(40 ; 5,8 ; 0,1)$ which is resolvable, using Theorem 12 , we get $N(39) \geqq 3$, whereas $n(39)=2$. Similarly from $\operatorname{SRGD}(136 ; 8,17 ; 0,1)$ we get $N(135) \geqq 6$, whereas $n(135)=4$.

ExAmple $(16)$. From $\operatorname{SRGD}(144 ; 9,16 ; 0,1)$ and Theorem 13 , we get $N(145) \geqq 7$, whereas $n(145)=4$.

Example (17). From $\operatorname{SRGD}(77 ; 7,11 ; 0,1)$ and Theorem 14 with $x=5$ and 9 respectively, we get $N(82) \geqq 3, N(86) \geqq 5$, whereas $n(82)=n(86)=1$.

9. Table of lower bounds of $N(v)$ for $v \leqq 150$. We provide below a table of lower bounds (1.b.) of $N(v)$ for those values of $v \leqq 150$, where the lower bound exceeds $n(v)$. In the last column under remarks we indicate how the corresponding lower bound was obtained. The numbers for which $v \equiv 2(\bmod 4)$ are starred.

10. Concluding remarks. We are conscious of the fact that the table given in the last section may be incomplete. There may be other values of $v$ for which $N(v)>n(v)$, as the pairwise balanced designs of index unity have not been exhaustively studied. Indeed they were improvised for the purposes of the present paper, though we hope that they may be useful in other combinatorial problems. Only a lower bound for $N(v)$ has been given and it may be possible to improve it in many cases.

Many new interesting problems arise as a result of the present work. A positive integer $n$ may be called an Eulerian number if there do not exist more than one orthogonal Latin square of order $n$. The density and distribution of Eulerian numbers is a question of great interest. The table of the last section shows that there exists a run of 24 consecutive non-Eulerian numbers from 63 to 86 . We shall show in a later communication by extending the methods of $\$ 7$, that there exist arbitrarily large runs of consecutive nonEulerian numbers. In other words there exist arbitrarily large gaps between consecutive Eulerian numbers.

It may also be observed that analogous methods for constructing orthogonal arrays of strength two and index $\lambda$ can be made to depend on pairwise balanced designs of index $\lambda$, which are defined as in $\$ 3$, except that every pair of distinct treatments occurs in $\lambda$ different blocks. It is hoped to consider these and allied problems in a subsequent communication. 


\begin{tabular}{|c|c|c|}
\hline$\pi$ & 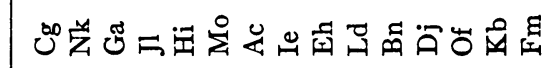 & 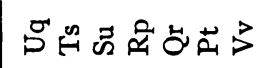 \\
\hline$\vec{\sim}$ & 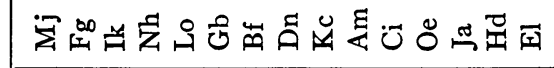 & 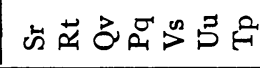 \\
\hline \& & 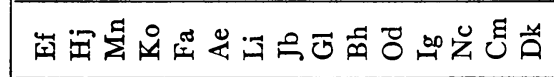 & 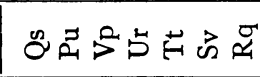 \\
\hline 9 & 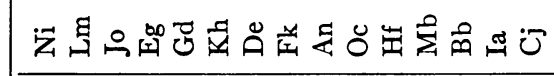 & 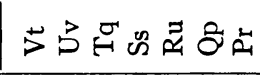 \\
\hline$\stackrel{\infty}{\sim}$ & 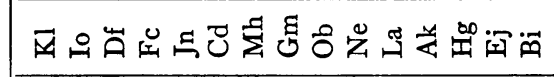 & 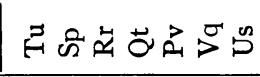 \\
\hline$\approx$ & 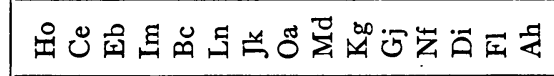 & 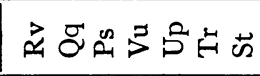 \\
\hline - & 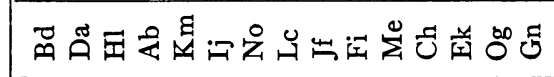 & 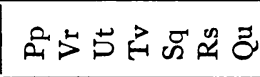 \\
\hline 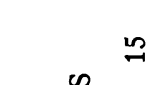 & 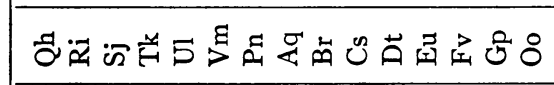 & 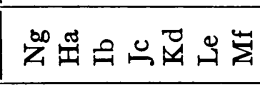 \\
\hline 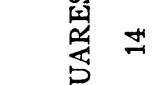 & 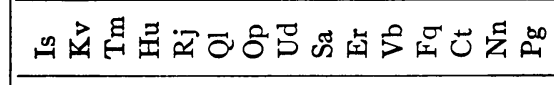 & 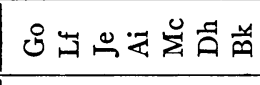 \\
\hline$m$ & 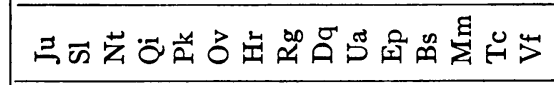 & 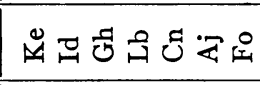 \\
\hline$\approx$ & 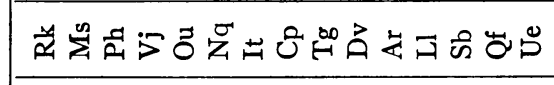 & 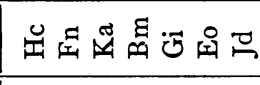 \\
\hline$\Rightarrow$ & 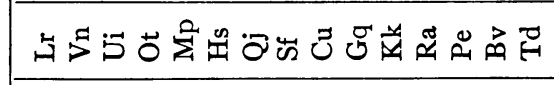 & 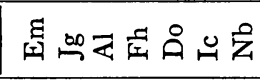 \\
\hline 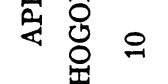 & 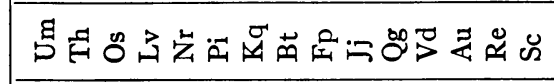 & 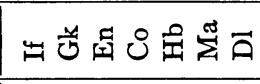 \\
\hline 案 & 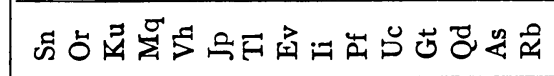 & 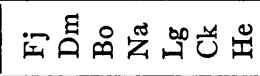 \\
\hline 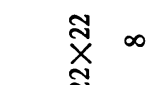 & 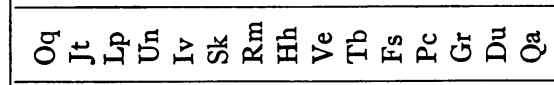 & 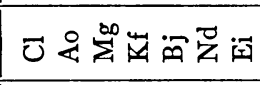 \\
\hline 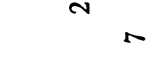 & 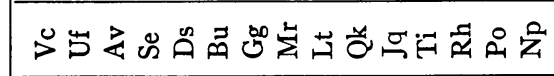 & 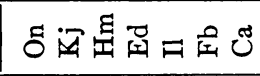 \\
\hline 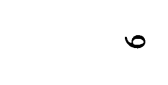 & 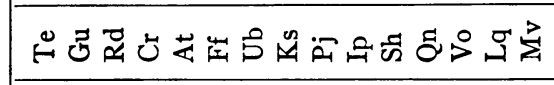 & 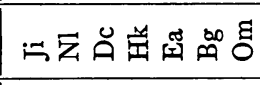 \\
\hline is & 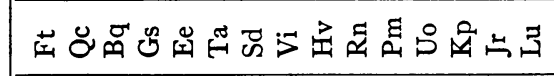 & 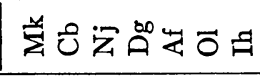 \\
\hline 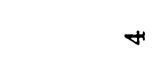 & 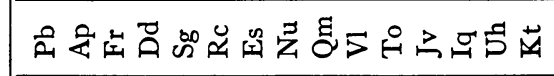 & 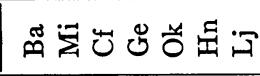 \\
\hline m & 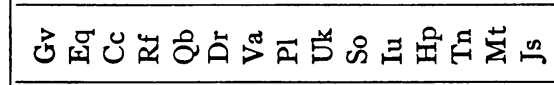 & 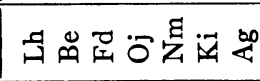 \\
\hline a & 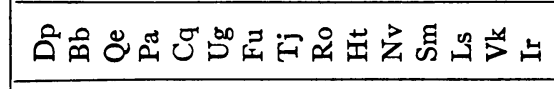 & 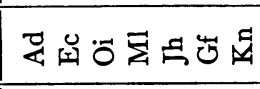 \\
\hline - & 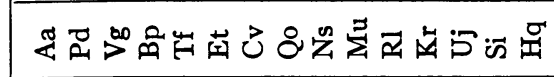 & 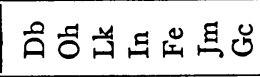 \\
\hline
\end{tabular}

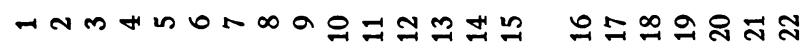




\section{BIBLIOGRAPHY}

1. W. W. R. Ball, Mathematical recreations and essays, revised by H. S. M. Coxeter, London Macmillan and Co. Ltd., 1942.

2. R. C. Bose, On the construction of balanced incomplete block designs, Ann. of Eugen. London vol. 9 (1939) pp. 353-399.

3. - - A note on the resolvability of balanced incomplete block designs, Sankhyā vol. 6 (1942) pp. 105-110.

4. - Mathematical theory of the symmetrical factorial design, Sankhyā vol. 8 (1947) pp. $107-166$.

5. - A note on orthogonal arrays, Ann. Math. Statist. vol. 21 (1950) pp. 304-305 (abstract).

6. - On the application of finite projective geometry for deriving a certain series of balanced Kirkman arrangements, Bull. Calcutta Math. Soc. Silver Jubilee vol. 51 (1959).

7. R. C. Bose and W. S. Connor, Combinatorial properties of group divisible incomplete block designs, Ann. Math. Statist. vol. 23 (1952) pp. 367-383.

8. R. C. Bose, S. S. Shrikhande and K. N. Bhattacharya, On the construction of group divisible incomplete block designs, Ann. Math. Statist. vol. 24 (1953) pp. 167-195.

9. R. C. Bose and S. S. Shrikhande, On the falsity of Euler's conjecture about the non-existence of two orthogonal Latin squares of order $4 t+2$, Proc. Nat. Acad. Sci. U.S.A. vol. 45 (1959) pp. 734-737.

10. K. A. Bush, A generalization of a theorem due to MacNeish, Ann. Math. Statist. vol. 23 (1952) pp. 293-295. 434.

11. - Orthogonal arrays of index unity, Ann. Math. Statist. vol. 23 (1952) pp. 426-

12. O. Eckenstein, Bibliography of Kirkman's school girl problem, Messenger of Math. vol. 41 (1911-1912) pp. 33-36.

13. L. Euler, Recherches sur une nouvelle espéce des quarres magiques, Verh. zeeuwsch Genoot. Wetenschappen vol. 9 (1782) pp. 85-239.

14. F. W. Levi, Finite geometrical systems, University of Calcutta, 1942.

15. H. F. MacNeish, Das problem der 36 offiziere, Jber. Deutsch. Math. Verein. vol. 30 (1921) pp. 151-153.

16. - Euler's squares, Ann. of Math. vol. 23 (1922) pp. 221-227.

17. H. B. Mann, The construction of orthogonal Latin squares, Ann. Math. Statist. vol. 13 (1942) pp. 418-423.

18. E. T. Parker, Construction of some sets of pairwise orthogonal Latin squares, Abstract 553-67, Notices Amer. Math. Soc. vol. 5 (1958) p. 815.

19. J. Peterson, Les 36 officers, Ann. of Math. (1901-1902) pp. 413-427.

20. P. Wernicke, Das problem der 36 offiziere, Jber. Deutsch. Math. Verein. vol. 19 (1910) pp. 264-267. 140.

21. F. Yates, Incomplete randomized blocks, Ann. of Eugen. London vol. 7 (1936) pp. 121-

University of North Carolina, Chapel Hill, North Carolina 